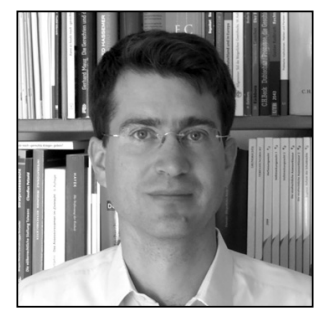

Christoph Conrad Henke

Dr. jur., LL.M.

\title{
Warum wir an der Universalität der westlichen Werte festhalten sollten
}

\section{Ausgangssituation ${ }^{*}$}

Die westlichen Werte lassen sich - soweit sie fundamentale Prinzipien der Gerechtigkeit betreffen - in vier Kategorien untergliedern: Gewaltverbot, Demokratie, Rechtsstaat und Menschenrechte. Der Westen hat diese Werte weitgehend in geltendes Recht umgesetzt. Er erhebt in Bezug auf sie Anspruch auf Universalität. ${ }^{{ }_{2}}$ Der weltweiten Verbreitung der übrigen Ideale stehen jedoch Hindernisse entgegen, wie folgende Beispiele aufzeigen:

Verschiedene Staaten und Autoren ziehen die Universalität der westlichen Werte in Zweifel. So argumentiert die VR China, die Menschenrechte seien „nicht nur historisch ein Produkt der europäisch-amerikanischen Kultur, sondern auch in Inhalt, Form und rechtlicher Ausgestaltung nur in diesen Kulturen beheimatet, gegenüber den asiatischen Kulturen hingegen seien sie in wichtigen Hinsichten fremd und unangemessen. Eben deshalb komme ihnen auch dort nur eine beschränkte Geltung zu, und die Allgemeine Erklärung der Menschenrechte sei durch eine asiatische Variante, wie es ja auch schon eine europäische gebe, zu ersetzen."* 3

Selbst in der deutschen Öffentlichkeit trifft die Anwendung rechtsstaatlicher Prinzipien gelegentlich auf Unverständnis. Dies zeigt der Fall Gäfgen. Die Polizei hatte Gäfgen Folter für den Fall angedroht, dass er den Aufenthaltsort des von ihm entführten Kindes nicht preisgebe. Daraufhin brachte Gäfgen die Polizei

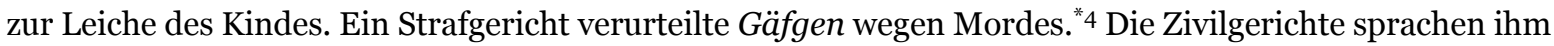
unter Berücksichtigung der Rechtsprechung des Europäischen Gerichtshofs für Menschenrechte (EGMR) ${ }^{*} 5$

1 Der Beitrag entwickelt Überlegungen fort aus Henke, Christoph, Zur Finalität des Völkerrechts. Eine völkerrechtliche Agenda, Münster u.a. 2012. Im dortigen Beitrag stehen konkrete völkerrechtliche Handlungsvorschläge im Vordergrund. Vorliegend geht es um die Herleitung fundamentaler Gerechtigkeitsprinzipien. Zwangsläufig kommt es insbesondere in Ziff. 1 bis 5 dieses Beitrags zu inhaltlichen Überschneidungen.

2 Vgl. zum Begriff der Universalität Bielefeldt, Heiner, Menschenrechtlicher Universalismus ohne eurozentrische Verkürzung. Zur Verständigung über die Universalität der Menschenrechte, in: Nooke, Günter/Lohmann, Georg/Wahlers, Gerhard, Gelten Menschenrechte universal? Freiburg 2008, S. $98 \mathrm{ff}$.

3 Zitiert nach Lohmann, Georg, Zur Verständigung über die Universalität der Menschenrechte, in: Nooke, Günter/Lohmann, Georg/Wahlers, Gerhard, Gelten Menschenrechte universal? Freiburg 2008, S. 47.

4 LG Frankfurt a.M., Urteil vom 28.7.2003, 5/22 Ks 2/o3 3490 Js 230118/o2; BGH, Entscheidung vom 21.5.2004, 2 StR 35/04.

5 EGMR, Gäfgen v. Deutschland, Entscheidung vom 1.6.2010, Nr. 22978/05. 
eine Entschädigung zu. ${ }^{* 6}$ Zahlreiche deutsche Bürger konnten nicht nachvollziehen, weshalb ein Mörder eine Entschädigung erhalten müsse. ${ }^{*} 7$

Gelegentlich gehen Angriffe auf westliche Werte von westlichen Staaten selbst aus. Dafür steht der Irak-Krieg von 2003, den die Mehrheit der Völkerrechtler für rechtswidrig erachtete, ${ }^{* 8}$ den der ehemalige UN-Generalsekretär Annan als illegalen Akt bezeichnete ${ }^{*} 9$ und gegen den das Bundesverwaltungsgericht „gravierende völkerrechtliche Bedenken” erhob. ${ }^{* 10} \mathrm{Zu}$ keinem Zeitpunkt zogen die westlichen Staaten eine strafrechtliche Verfolgung der Verantwortlichen in Erwägung. Im Gegenteil, sie übertrugen letzteren nach deren Ausscheiden aus dem Amt zum Teil politische Aufgaben anderer Art. So bestellten sie Blair zum Sondergesandten des Nahostquartetts, bestehend aus den USA, der EU, Russland und den Vereinten Nationen. ${ }^{* 11}$

Wer die weltweite Verbreitung von Gewaltverbot, Demokratie, Rechtsstaat und Menschenrechten anstrebt, sollte eine überzeugende Begründung vorweisen können. Der vorliegende Beitrag versucht, die Grundlagen westlicher Werte herauszustellen.

\section{Westliche Werte}

Die fundamentalen Gerechtigkeitsprinzipien, die alle Staaten verwirklichen sollten, sind teils völkerrechtlich verbindlich festgeschrieben, teils als Zielvorgaben ausformuliert:

So ist in Art. 2 Ziff. 4 UN-Charta ${ }^{* 12}$ das völkerrechtliche Gewaltverbot geregelt. Danach ist die Anwendung von Gewalt gegenüber einem anderen Staat regelmäßig unzulässig. Ausnahmen betreffen individuelle Selbstverteidigung (Notwehr), ${ }^{*}{ }^{3}$ kollektive Selbstverteidigung (Nothilfe) ${ }^{*} 14$ sowie die Autorisierung eines Einsatzes durch den UN-Sicherheitsrat. ${ }^{*} 15$

$\mathrm{Zu}$ den grundlegenden Gerechtigkeitsprinzipien gehört ein Mindeststandard an Menschenrechten: Vom zwingenden Völkerrecht (ius cogens), das von allen Staaten einzuhalten ist, sind untersagt: Völkermord, ${ }^{* 16}$ Folter, Sklavenhandel und willkürliche Tötung. ${ }^{*}{ }^{17}$ Ein Teil der Staatengemeinschaft hat wichtige Menschenrechte im Internationalen Pakt über wirtschaftliche, soziale und kulturelle Rechte (UN-Sozialpakt) ${ }^{* 18}$ sowie im Internationalen Pakt über bürgerliche und politische Rechte nebst Fakultativprotokollen (UNZivilpakt) ${ }^{* 19}$ vom 16. Dezember 1966 rechtsverbindlich festgeschrieben. Auf regionaler Ebene ist die Europäische Menschenrechtskonvention (EMRK) ${ }^{* 20}$ mit ihren Individualbeschwerdemöglichkeiten am weitesten entwickelt. Die Allgemeine Erklärung der Menschenrechte (AEMR), ${ }^{*}{ }^{21}$ die ohne Gegenstimmen von der UN-Generalversammlung verabschiedet und in der Millenniums-Erklärung ${ }^{* 22}$ bekräftigt wurde, legt über das geltende Recht hinaus für alle Staaten menschenrechtliche Ziele fest, die auf längere Sicht verwirklicht werden sollen. Sie enthält „das von allen Völkern und Nationen zu erreichende gemeinsame Ideal” und beinhaltet u.a. das Recht auf Leben, Heirat, Eigentum, Religionsfreiheit, Versammlungsfreiheit, Bildung und Arbeit sowie das Verbot von Sklaverei und Folter.

6 LG Frankfurt a.M., Urteil vom 4.8.2011, 2-04 O 521/o5; OLG Frankfurt a.M., Urteil vom 10.10.2012, 1 U 201/11.

Stern, Gäfgens Entschädigung sorgt für Empörung, 4.8.2011, www.stern.de (Stand: 4.6.2012).

8 Jahn, Egbert, Politische Streitfragen, Bd. 3, Internationale Politik, Wiesbaden 2012, S. 195. - DOI: http://dx.doi. org/10.1007/978-3-531-94313-8

9 FAZ, Kofi Annan nennt Irak-Krieg ,illegal”, 16.9.2004, www.faz.de (Stand: 4.6.2012).

1o BVerwGE 127, 302, 353.

FAZ, Blair und das Nahost-Quartett, 28.6.2007, www.faz.de (Stand: 4.6.2012).

Charta der Vereinten Nationen vom 26.6.1945, BGBl. 1973 II S. 430.

Art. 51 UN-Charta.

Art. 51 UN-Charta.

Kapitel VII UN-Charta.

BVerfG, Beschluss vom 12.12.2000, 2 BvR 1290/99.

Stein, Torsten/v.Buttlar, Christian, Völkerrecht, 13. Aufl., München 2012, Rn. 1000.

Internationaler Pakt über wirtschaftliche, soziale und kulturelle Rechte vom 19.12.1966; BGBl. 1973 II S. 1569.

Internationaler Pakt über bürgerliche und politische Rechte vom 19.12.1966; BGBl. 1973 II S. 1533.

Europäische Konvention zum Schutz der Menschenrechte und Grundfreiheiten vom 4.11.1950; BGBl. 1952 II S. 685.

Resolution der Generalversammlung vom 10.12.1948, A/RES/217 III A.

United Nations Millennium Declaration, Resolution der Generalversammlung vom 8.9.200o, A/RES/55/2, Ziff. 25. 
Aus der AEMR ergibt sich ein weltweit anzustrebender demokratischer Mindeststandard. So heißt es in Art. 21 AEMR: „Jeder hat das Recht, an der Gestaltung der öffentlichen Angelegenheiten seines Landes unmittelbar oder durch frei gewählte Vertreter mitzuwirken... Der Wille des Volkes bildet die Grundlage für die Autorität der öffentlichen Gewalt; dieser Wille muss durch regelmäßige, unverfälschte, allgemeine und gleiche Wahlen mit geheimer Stimmabgabe oder einem gleichwertigen freien Wahlverfahren zum Ausdruck kommen."

Schließlich gehört zu den fundamentalen Gerechtigkeitsprinzipien ein anzustrebender Mindeststandard an Rechtsstaatlichkeit. Dementsprechend ist in Art. 10 AEMR verankert: „Jeder hat bei der Feststellung seiner Rechte und Pflichten sowie bei einer gegen ihn erhobenen strafrechtlichen Beschuldigung in voller Gleichheit Anspruch auf ein gerechtes und öffentliches Verfahren vor einem unabhängigen und unparteiischen Gericht."

Ein Ansatz zur Begründung dieser westlichen Werte sollte idealerweise erläutern, weshalb es sich um weltweit einzuführende Gerechtigkeitsprinzipien handelt, wie die jeweiligen Prinzipien in Konfliktfällen zu gewichten sind und unter welchen Voraussetzungen Gerechtigkeitsprinzipien zum Katalog hinzutreten oder aus ihm herausfallen können.

\section{Umsetzung}

Gegen eine Universalität der westlichen Werte könnte man einwenden, dass diese im Detail umstritten sind. Hieran anknüpfend könnte man in Zweifel ziehen, ob eine weltweite Umsetzung faktisch möglich wäre. In der Staatengemeinschaft besteht keine Einigkeit über die Auslegung der vorerwähnten Prinzipien. ${ }^{* 23}$ Vielmehr gibt es eine an „Widersprüchen und Konflikten reiche Vielfalt von Traditionen”.*24

Dies muss, wie ein Vergleich mit innerstaatlichem Verfassungsrecht zeigt, kein unüberwindbares Hindernis bei der Verwirklichung einer gemeinsamen Rechtsordnung sein. Auf nationaler Ebene erfolgt die Konkretisierung der abstrakten Verfassungsvorschriften im Wege sog. praktischer Konkordanz: ${ }^{*} 5$ Gesetzgeber und Gerichte haben unterschiedliche Rechtsgüter, die untereinander in Konflikt geraten, auszubalancieren und die Grenzlinien zwischen ihnen zu bestimmen, um ihnen zu größtmöglicher Wirksamkeit zu verhelfen. So hatte das Bundesverfassungsgericht anlässlich der Streitigkeiten um die Aussage „Soldaten sind Mörder"*26 Meinungsfreiheit und Ehrenschutz gegeneinander abzuwägen und im Wege praktischer Konkordanz eine Grenzlinie zwischen zulässiger und unzulässiger Äußerung zu ziehen. Ein Instrumentarium, Streitigkeiten über die Auslegung abstrakter Rechtsprinzipien beizulegen, existiert also vom Grundsatz her durchaus.

Nach dem Vorbild des innerstaatlichen Verfassungsrechts und des Schutzsystems der EMRK könnte auch die Umsetzung fundamentaler Gerechtigkeitsprinzipien auf internationaler Ebene erfolgen. Dies betrifft, da das Gewaltverbot bereits in geltendes Völkerrecht umgesetzt ist, in erster Linie die AEMR.

Idealiter enthalten die Verfassungen der Staaten die Regelungen der AEMR oder einen darüber hinausgehenden Schutzstandard. Dadurch kommt den Regelungen erhöhter Bestandsschutz zu. Die Regelungen der AEMR sind in einfachen Gesetzen zu konkretisieren. Exekutive und Rechtssubjekte wenden die entsprechenden Gesetze im Rahmen ihres Ermessensspielraums bzw. ihrer Vertragsfreiheit an. Die innerstaatlichen Instanzgerichte prüfen, ob Exekutive und Rechtssubjekte ihren (ersten) Entscheidungskorridor bei der Festlegung der Grenzlinien zwischen den einzelnen Rechtsgütern eingehalten haben. Soweit vorhanden prüft das Verfassungsgericht, ob die Instanzgerichte die Verfassungsvorgaben gewahrt haben. Dabei räumt es den Instanzgerichten einen (zweiten) Entscheidungskorridor ein.

Auf internationaler Ebene ist ein völkerrechtlich bindendes Vertragswerk zu schaffen, das die Regelungen der AEMR enthält und das alle Staaten ratifizieren. UN-Zivilpakt und UN-Sozialpakt können als Ausgangsbasis dienen. Parallel dazu ist den Rechtssubjekten - aus Gründen der Subsidiarität nach Ausschöpfung des innerstaatlichen Rechtswegs - nach dem Vorbild der EMRK die Möglichkeit der Individualbeschwerde gegen alle Staaten einzuräumen. Ein internationales, unabhängiges Gericht entscheidet, ob

23 Kempen, Bernhard/Hillgruber, Christian, Völkerrecht, München 2007, S. 332.

24 Albert, Hans, Die Idee der kritischen Vernunft. Zur Problematik der rationalen Begründung und des Dogmatismus, Aufklärung und Kritik 2/1994, S. $16 f f$.

25 Hesse, Konrad, Grundzüge des Verfassungsrechts der Bundesrepublik Deutschland, 20. Aufl., Heidelberg 1995, Rn. 317ff.

26 BVerfGE 93, $266 \mathrm{ff}$. 
die nationalen Gerichtsentscheidungen korrekt zustande gekommen sind und mit dem völkerrechtlichen Vertrag in Einklang stehen. Das internationale Gericht räumt den nationalen Verfassungsgerichten einen (dritten) Entscheidungskorridor ein. Nur wenn dieser verlassen wurde, liegt eine Vertragsverletzung vor. In ähnlicher Weise entscheidet der EGMR bereits gegenwärtig über Konventionsverletzungen. Insoweit hat er hervorgehoben, dass die Mitgliedstaaten der EMRK in vielen Fällen - etwa bei der Durchsuchung einer Wohnung - einen weiten Ermessenspielraum haben. ${ }^{*} 27$

Der dreifach gestaffelte und auf internationaler Ebene besonders breite Entscheidungskorridor sorgt dafür, universale Prinzipien und kulturelle Unterschiede miteinander in Einklang zu bringen. Der Verlauf des Korridors ist den völkerrechtlichen Verträgen nicht in allen Einzelheiten zu entnehmen. Doch ist er keineswegs willkürlich. Viele Entscheidungen sind prognostizierbar. So würde ein nach dem Vorbild des EGMR geschaffenes internationales Gericht aller Voraussicht nach wie dieser entscheiden, dass Folterandrohung - wie im Fall Gäfgen - unzulässig ist, während Entscheidungen zu Kruzifixen in Klassenzimmern $^{* 28}$ oder zu Kopftuchverboten an Universitäten ${ }^{* 29}$ sich innerhalb des Entscheidungskorridors der Staaten bewegen.

\section{Letztbegründung}

Ob und wie sich ein Allgemeinanspruch der westlichen Werte begründen lässt, ist eine metaethische Fragestellung. Die Metaethik befasst sich aus Sicht eines externen Beobachters mit normativen Aussagen, d.h. mit Aussagen nicht über das Sein, sondern über das Sollen. Sie geht Fragen nach wie: Was sind normative Aussagen? Gibt es normative Tatsachen? Können normative Aussagen wahr sein? Warum handeln Menschen moralisch? Wie lassen sich normative Aussagen rechtfertigen? ${ }^{*} 30$ Die letztgenannte Frage steht im Mittelpunkt dieser Erörterung. Falls eine Rechtfertigung normativer Aussagen von vornherein ausgeschlossen sein sollte, lassen sich auch normative Aussagen zur Universalität der westlichen Werte nicht überzeugend rechtfertigen. Soweit es metaethische Vorgaben für eine Begründung der Universalität westlicher Werte geben sollte, wären diese zu berücksichtigen. In diesem Zusammenhang lässt sich zwischen normativen Aussagen einerseits (Gerechtigkeitsprinzipien, weltweit einzuführenden Rechtsprinzipien) und deskriptiven Aussagen andererseits (geltendem Recht) unterscheiden. Vor allem zwei Problemkreise erschweren eine Rechtfertigung normativer Aussagen.

\section{a) Unzulässigkeit eines Schlusses vom Sein auf das Sollen}

Nach überwiegender Ansicht ist ein logisch korrekter Schluss vom Sein auf das Sollen nicht möglich (Humes Gesetz). ${ }^{*}{ }^{1}$ Die Bedeutung der Trennung von Sein und Sollen haben in Bezug auf das Recht u.a. Kelsen, ${ }^{*}{ }^{2}$ H.L.A. Hart ${ }^{*} 33$ und Luhmann ${ }^{*} 34$ hervorgehoben. Nach ihnen kann vom Ist-Zustand des Rechts oder sonstigen Seins-Tatsachen nicht auf dessen Soll-Zustand geschlossen werden. Aus der Existenz des völkerrechtlichen ius cogens (etwa des Verbots des Völkermordes) kann nicht geschlussfolgert werden, dass das ius cogens gerecht ist. Aus dem Umstand, dass Menschen in einer Demokratie ggf. zufriedener sind als in einer Diktatur (Sein), kann nicht hergeleitet werden, dass Demokratie eingeführt werden soll (Sollen). Ein Konsens der Staatengemeinschaft über einen Mindeststandard an Menschenrechten (Sein) bedeutet deshalb nicht zwangsläufig, dass der Mindeststandard etwaigen Alternativen vorzuziehen wäre (Sollen).

\footnotetext{
EGMR, Buck v. Germany, Entscheidung vom 28.4.2005, Nr. 41604/98, § 44.

EGMR, Lautsi u.a.v. Italien, Entscheidung vom 18.3.2011, Nr. 30814/06.

EGMR, Sahin v. Türkei, Entscheidung vom 10.11.2005, Nr. 44774/98.

3о Die Informationen zur Metaethik wurden übernommen aus Scarano, Nico, Metaethik. Ein systematischer Überblick, in: Düwell, Marcus/Hübenthal, Christoph/Werner, Micha (Hrsg.), Handbuch Ethik, Stuttgart, Weimar, 2002, S. 25ff.

31 Hepfer, Karl, Philosophische Ethik, Freiburg 2008, S. 24; vgl. Hume, David, A Treatise of Human Nature (Buch III, Teil I, Kapitel I), zitiert nach Hepfer, Karl, Philosophische Ethik, Freiburg 2008, S. 24.

32 Jestaedt, Matthias (Hrsg.), Kelsen, Hans, Reine Rechtslehre, 1934 (Studienausgabe), Tübingen 2008.

33 Hart, H.L.A., Der Begriff des Rechts, Frankfurt a.M. 1973, S. 255.

34 Luhmann, Niklas, Das Recht der Gesellschaft, Frankfurt a.M. 1995, S. 78.
} 


\section{b) Münchhausen-Trilemma}

Nach verbreiteter, im sog. Münchhausen-Trilemma zum Ausdruck kommender Ansicht kann es keine überzeugende Letztbegründung geben. Dies postulieren etwa Albert ${ }^{*} 35$ und andere Vertreter des von Popper begründeten Kritischen Rationalismus. Danach führen Letztbegründungsversuche im Ergebnis (1) zu einem Dogma, das sich nicht beweisen lässt, (2) zu einem infiniten Regress, bei dem sich jede Begründung wieder hinterfragen lässt oder (3) zu einem Zirkelschluss. ${ }^{*}{ }^{6}$ Unbezweifelbare Erkenntnisse gebe es nicht. Einen ähnlichen Ansatz vertrat in der Antike Agrippa. ${ }^{*} 37$ Um nicht in einen Widerspruch zu geraten, behaupten die Vertreter des Kritischen Rationalismus nicht die Erwiesenheit des Münchhausen-Trilemmas. Sie betrachten dieses vielmehr als philosophische Hypothese. ${ }^{*} 38$ Auf die Universalität westlicher Werte übertragen, bedeutet dies, dass eine Letztbegründung nicht möglich ist.

Indes lässt das Münchhausen-Trilemma zwei andere Begründungswege zu: Zum einen lassen sich westliche Werte möglicherweise auf ein Dogma (eine Prämisse) zurückführen, dem der Argumentationspartner zustimmen kann. Damit sind zwar nicht sämtliche Zweifel an der Richtigkeit ausgeschlossen. Doch liegt eine hinreichende Begründung für diejenigen vor, die die Prämisse akzeptieren. Zum anderen geht der Kritische Rationalismus davon aus, dass Normen von Menschen gemacht sind. ${ }^{*} 39$ Sie dienen dazu, Probleme zu lösen. Der Gesetzgeber kann Alternativen vergleichen. Bewähren sich Normen, kann er sie beibehalten, andernfalls modifizieren. Die Kriterien, nach denen sich dies beurteilt, sind nach dem Kritischen Rationalismus von Menschen festzulegen. In der Entwicklung solcher Kriterien könnte ein zweiter Ansatz zur Rechtfertigung der Universalität westlicher Werte zu sehen sein.

\section{Klassische Begründungen}

Einzugehen ist noch auf drei klassische Begründungsansätze, die nicht zu überzeugen vermögen.

\section{a) Naturrecht}

Nach bestimmter Auffassung lassen sich Gerechtigkeitsprinzipien ${ }^{*} 40$ oder Teile des geltenden Rechts aus der Natur bzw. der Vernunft des Menschen herleiten.

Der Naturrechtsgedanke erlebte unter dem Eindruck des Zweiten Weltkriegs einen Aufschwung, der in der Radbruchschen Formel zum Ausdruck kam. ${ }^{* 11}$ Der Naturrechtsgedanke hat Eingang in das positive Recht gefunden. So stellt Art. 20 Abs. 3 GG klar, dass Gesetz und Recht voneinander abweichen können, und erteilt der Argumentation „Gesetz ist Gesetz”"42 eine Absage. Art. 51 UN-Charta hebt das „naturgegebene" Recht zur Selbstverteidigung hervor. Dem Naturrecht ist zu verdanken, dass Autoren wie Thomas v.Aquin, Grotius, Wolff oder Kant elementare Rechtsgrundsätze herausarbeiteten (argumentum ad verecundiam), dies eine lange Tradition bewahrte (historisches Argument) und in völkerrechtliche Texte wie UN-Charta oder AEMR einfloss.

Wenn der Naturrechtsgedanke zum Zweck der Letztbegründung herangezogen wird, vermag er nicht zu überzeugen. Er zieht einen unzulässigen Schluss vom Sein (Natur, Vernunft) auf das Sollen. Doch „von dem, was ist oder tatsächlich geschieht, kann kein Schluss auf das, was sein oder geschehen soll" gezogen

\footnotetext{
Albert, Hans, Traktat über kritische Vernunft, 4. Aufl., Tübingen 1980, S. $11 \mathrm{ff}$.

Albert, Hans, Traktat über kritische Vernunft, 4. Aufl., Tübingen 1980, 13.

Reich, Klaus (Hrsg.), Diogenes Laertius, Leben und Meinungen berühmter Philosophen, Bd. 2, Hamburg 2008, 9. Buch, 88.

Albert, Hans, Traktat über kritische Vernunft, 4. Aufl., Tübingen 1980, S. 187.

39 Die Informationen in diesem Absatz wurden übernommen von Niemann, Hans-Joachim, Wie objektiv kann Ethik sein, Aufklärung und Kritik, Sonderheft 5/2001, S. 23.

40 Vgl. Kelsen, Hans, Was ist Gerechtigkeit, Stuttgart 2000, S. 46.

41 Radbruch, Gustav, Gesetzliches Unrecht und übergesetzliches Recht, Süddeutsche Juristenzeitung 1946, S. 105,107 (Rechtschreibung angepasst).

42 Vgl. zur Aussage „Gesetz ist Gesetz”, die den deutschen Juristenstand gegenüber dem NS-Unrecht wehrlos gemacht habe, Radbruch, Gustav, Gesetzliches Unrecht und übergesetzliches Recht, Süddeutsche Juristenzeitung 1946, S. 105.
} 
werden. ${ }^{*} 43$ Ohne Zusatzprämisse ist ein naturrechtlicher Ansatz zum Scheitern verurteilt. Mit einer Naturrechtslehre, die dies nicht berücksichtigt, lässt sich „alles und daher nichts” beweisen. ${ }^{*} 4$

\section{b) Utilitarismus}

Der von Autoren wie Bentham und J.S. Mill entwickelte Utilitarismus lenkt den Blick darauf, Politik nicht am Wohl der herrschenden Klasse, sondern am Allgemeinwohl auszurichten. Dies kommt in dem Schlagwort des größten Glücks der größten Zahl bzw. dem Ziel „die Totalsumme des Wohlseins der Individuen zu vermehren" 45 zum Ausdruck. Insoweit lässt sich argumentieren, eine Verwirklichung der westlichen Werte erhöhe das Wohl der Bevölkerung. In diesem Sinne kann eine Zunahme demokratischer Partizipation empirischen Untersuchungen zufolge das Glücksniveau der Bevölkerung erhöhen. ${ }^{*} 46$ Dieser Aspekt des Utilitarismus kann in die Rechtfertigung fundamentaler Gerechtigkeitsprinzipien einfließen.

Doch gerät der Utilitarismus aus strukturellen Gründen mit ihnen immer wieder in Konflikt. So vertritt Hare die Ansicht, ein noch nicht gezeugter Mensch habe das gleiche Lebensrecht wie ein geborener Mensch. Dementsprechend dürfe man ein neu geborenes behindertes Kind töten, um einem noch nicht gezeugten gesunden und voraussichtlich glücklicheren Menschen die Möglichkeit zum Leben zu verschaffen. ${ }^{*} 47$ In ähnlichem Sinne argumentiert Singer. ${ }^{*}{ }^{48}$ Solche Ansichten stehen in Widerspruch u.a. zu Strafrecht, Grundgesetz, Europäischer Grundrechtecharta, Europäischer Menschenrechtskonvention und AEMR.

\section{c) Gesellschaftsvertrag}

Ein weiterer Ansatz ist die Rückführung von Gerechtigkeitsprinzipien auf einen Gesellschaftsvertrag. So leitet Rawls ${ }^{*} 49$ in Fortentwicklung von Überlegungen Lockes, Rousseaus und Kants Gerechtigkeitsgrundsätze aus einer unter dem „Schleier des Nichtwissens” zustande kommenden fiktiven Übereinkunft her. Dieser Ansatz lenkt den Blick auf den mutmaßlichen Willen der Rechtssubjekte.

Indes liefert auch er keine überzeugende Letztbegründung fundamentaler Gerechtigkeitsprinzipien. So bleibt die Frage, weshalb ein fiktiver Gesellschaftsvertrag als Rechtfertigung genügen sollte. Außerdem ist Konsens in einer größeren Gruppe kaum zu erzielen. Dies wirft u.a. die weitere Frage auf, ob eine (fiktiv) überstimmte Minderheit an den Gesellschaftsvertrag gebunden ist und wie ihre Rechte geschützt werden. Zudem tritt das Problem auf, weshalb die Abstimmenden im Nachhinein an den Vertrag gebunden sein sollten, während tradierte Regelsysteme eine solche Bindung tendenziell ablehnen (Wegfall der Geschäftsgrundlage, clausula rebus sic stantibus). ${ }^{*}{ }^{\circ}$ Die Antworten auf diese Fragen lassen sich wiederum hinterfragen, was letztlich zu einem infiniten Regress bzw. Dogma im Sinne des Münchhausen-Trilemma führt. ${ }^{*}{ }^{{ }_{1}}$

Im Übrigen ist zweifelhaft, ob die Ergebnisse eines unter dem Schleier des Nichtwissens zustande gekommenen Gesellschaftsvertrags stets wünschenswert sind. In der Praxis dürften Rawls ' Gedankenexperimente den Anfang des 18. Jahrhunderts geschlossenen Piraterie-Verträgen nahe kommen. Diese sahen nicht selten vor, dass die Piraten eines Schiffes ihren Kapitän demokratisch wählten und im Falle von Verletzungen, die sie wegen des Schleiers des Nichtwissens vorab nicht voraussehen konnten, eine Invalidenrente erhielten. ${ }^{*}{ }^{2}$ Was diesen Verträgen u.a. fehlte, war das Verbot von Gewaltanwendung gegenüber Dritten.

43 Kelsen, Hans, Was ist Gerechtigkeit, Stuttgart 2000, S. 47. Interpunktion teilweise geändert.

44 Kelsen, Hans, Was ist Gerechtigkeit, Stuttgart 2000, S. 48.

45 Bentham, Jeremy, Principles of Morals and Legislation, S. 5; zitiert nach Dumont, Etienne (Hrsg.), Jeremias Bentham's Principien der Gesetzgebung, Nachdruck 1966.

46 Frey, Bruno S./Stutzer, Alois, Happiness, Economy and Institutions, in: The Economic Journal, 110 (466, Oktober), 2000, S. 918ff.; zitiert nach http://e-collection.library.ethz.ch (Stand: 18.7.2012). - DOI: http://dx.doi.org/10.1111/1468-0297.00570.

47 Hare, Richard Mervyn, Das missgebildete Kind. Moralische Dilemmata für Ärzte und Eltern, in: Leist, Anton (Hrsg.), Um Leben und Tod, Frankfurt a.M. 1990, S. 374, 382.

48 Singer, Peter, Praktische Ethik, Stuttgart 1984, S. $200 \mathrm{ff}$. und 223.

49 Rawls, John, Eine Theorie der Gerechtigkeit, Frankfurt a.M. 1975, S. $28 f$.

50 Vgl. § 313 BGB.

51 Vgl. Radnitzky, Gerard, Zeitgenössische Gerechtigkeitstheorien unter der logischen Lupe, Aufklärung und Kritik, Sonderheft 2/1996, S. 31ff.

52 Speckmann, Thomas, Teuflische Kostümierungen der Egalität. Piraten als Demokraten in einem undemokratischen Zeitalter, FAZ, 28.1.2009, Nr. 23, S. N3. 
Die Piraten beschränkten sich nicht darauf, als Ackerbauern und Viehzüchter Inseln zu kolonialisieren; sie spezialisierten sich auf Raubzüge.

\section{Herleitung aus Prämissen}

Da eine Letztbegründung nicht möglich ist, verbleibt gemäß dem Münchhausen-Trilemma die gedankliche Rückführung auf eine Prämisse (ein Dogma). Macht man diesen Schritt mit, könnte eine Ausgangsbasis möglicherweise gefunden werden. $\mathrm{Zu}$ den Prämissen, auf welche sich die Staatengemeinschaft beruft, gehören Frieden, Konsens, Gleichheit, völkerrechtliche Verpflichtung und die Betrachtung der Menschenrechte als Dogma, das keiner weiteren Begründung bedarf.

\section{a) Frieden}

Das völkerrechtliche Gewaltverbot, Demokratie, Rechtsstaatlichkeit sowie ein Mindeststandard an Menschenrechten lassen sich nach übereinstimmender Ansicht der Staatengemeinschaft auf die Prämisse zurückführen, dass Frieden wünschenswert ist. ${ }^{*} 53$ Allerdings kann die gewaltsame Ausschaltung von Gegnern unter Verstoß gegen die genannten Grundsätze auch zu Frieden im Sinne einer „Friedhofsruhe” führen. Es erscheint auch wenig erfolgversprechend, allein mit Hilfe des Friedensziels kollidierende Rechtsgüter gegeneinander abzuwägen, um beispielsweise zu ermitteln, ob eine militärische Intervention zum Schutz der Menschenrechte gerechtfertigt ist.

\section{b) Konsens}

Ein Mindeststandard an grundlegenden Gerechtigkeitsprinzipien lässt sich möglicherweise auf einen Konsens der Staatengemeinschaft zurückführen. In diesem Sinne verweist die Präambel der AEMR auf „ein gemeinsames Verständnis dieser Rechte und Freiheiten”. Der Konsens der Staatengemeinschaft ist schwer zu erzielen und daher ein starkes Argument. Er liefert jedoch keine Gewissheit.

\section{c) Gleichheit}

Nach der Präambel der AEMR handeln die Staaten in Anerkennung „der gleichen und unveräußerlichen Rechte” aller Menschen. Art. 1 AEMR hebt hervor, dass alle Menschen „gleich an Rechten” geboren sind. Die Prämisse, dass alle Menschen im Rechtssinne gleichwertig sind und deshalb einen Mindestschutzstandard genießen sollten, leuchtet der überwiegenden Mehrheit der Menschen ein. Ergänzend lässt sich auf Rousseau verweisen. ${ }^{*} 54$ Doch bleibt die Frage, wie sich aus dem abstrakten Konzept der Gleichheit komplexe Regelungen zu Gewaltverbot, Demokratie, Rechtsstaatlichkeit und Menschenrechten herleiten lassen. Dies dürfte das Moment der Gleichheit überdehnen und zusätzliche Prämissen erfordern.

\section{d) Völkerrechtliche Verpflichtung}

Die Staatengemeinschaft stuft das Hinwirken auf grundlegende Gerechtigkeitsprinzipien als völkerrechtliche Verpflichtung ein. ${ }^{*} 5$ Die AEMR erging in Umsetzung einer solchen Verpflichtung. In diesem Sinne stellt die Präambel der AEMR klar, dass sich die Mitgliedstaaten „verpflichtet haben, in Zusammenarbeit mit den Vereinten Nationen auf die allgemeine Achtung und Einhaltung der Menschenrechte und Grundfreiheiten hinzuwirken.” Indes bleibt auch insoweit zu beachten: Wegen der Unzulässigkeit des Schlusses

\footnotetext{
Vgl. die Präambeln der AEMR und der UN-Charta.

54 Schmidts, Ludwig (Hrsg.), Rousseau, Jean-Jacques, Emil oder über die Erziehung, 13. Aufl., Paderborn 1998, S. 14: „In der natürlichen Ordnung sind alle Menschen gleich.”

55 Siehe auch Art. 1 Ziff. 3 UN-Charta.
} 
vom Sein auf das Sollen kann von einer rechtlichen Verpflichtung nicht auf das Sollen (Gerechtigkeit) geschlossen werden.

\section{e) Menschenrechte als Dogma}

Der Glaube an Gewaltverbot, Demokratie, Rechtsstaat und Menschenrechte lässt sich ggf. als Dogma im Sinne des Münchhausen-Trilemma ansehen, das keiner weiteren Begründung bedarf. So betont die Präambel der UN-Charta den „Glauben an die Grundrechte des Menschen.” Auch die Präambel der AEMR hebt den „Glauben an grundlegende Menschenrechte” hervor. Damit scheint es nach Ansicht der Staatengemeinschaft einen Mindeststandard zu geben, der so selbstverständlich ist, dass er keiner weiteren Rechtfertigung bedarf. Indes handelt es sich auch bei dieser Ansicht nicht um eine hinreichende Antwort auf die Frage nach der Rechtfertigung umfassender Grundsätze. Letztlich manifestiert sich in ihr eine Kapitulation vor dem Problem.

\section{Beweisproblematik}

Nachdem die Herleitung der westlichen Werte aus nur einem Prinzip bzw. nur einer Prämisse nicht möglich erscheint, ist nach einer komplexeren Lösung Ausschau zu halten. Diese könnte in der Verständigung auf Kriterien liegen, mit deren Hilfe sich feststellen lässt, welche Rechtsnorm gerecht ist. Es geht mit anderen Worten um den richtigen Beweismaßstab. Im Folgenden wird vorgeschlagen, zur Lösung des Problems Überlegungen aus dem Kritischen Rationalismus, den Tatsachenfeststellungen vor Gericht sowie dem juristisch/politologischen Legitimationskonzept miteinander zu verbinden.

\section{a) Fachspezifische Beweismaßstäbe}

Die Beweismaßstäbe in den jeweiligen Fachbereichen sind unterschiedlich. Ein besonders strenger Beweismaßstab gilt in der Erkenntnistheorie. Viele Autoren weisen darauf hin, dass Erkenntnis trügerisch sein könne. Kant etwa vertritt die Ansicht, die Sinne könnten nicht die „Dinge an sich”, sondern nur ihre Erscheinungen wahrnehmen. ${ }^{*}{ }^{6}$ Putnam hat die Frage ins Gespräch gebracht, ob wir möglicherweise ein „Gehirn in einem Tank" sein könnten, das an einen Computer angeschlossen ist, der eine Außenwelt vorspiegelt. ${ }^{*} 57$ Letztlich ist aus erkenntnistheoretischer Sicht wohl nicht zweifelsfrei zu beweisen, dass eine Außenwelt existiert, auch wenn es vernünftig ist, dies anzunehmen.

In der Mathematik gilt ebenfalls ein strenger Beweismaßstab. Mathematiker haben die für die Primzahlverteilung relevante Riemannsche Vermutung ${ }^{*}{ }^{*} 8$ auf den ersten 10 Billionen Positionen geprüft und für richtig befunden. ${ }^{*}{ }^{5}$ Dennoch ist der mathematische Beweis nicht geführt, denn bei der 11billionensten Position könnte eine Abweichung gegeben sein. Erst wenn eine Abweichung bei allen denkbaren Positionen ausgeschlossen ist, ist die Riemannsche Vermutung bewiesen. Nicht auseinandersetzen muss sich ein mathematischer Beweis mit der Frage, ob eine Außenwelt existiert.

In der Physik genügt ein gegenüber Erkenntnistheorie und Mathematik weniger strenger Beweismaßstab. So galt das Higgs-Bosons, nach dem die Teilchenphysiker lange Zeit fahndeten, als entdeckt, nachdem die Wahrscheinlichkeit eines Zufallsfundes auf ca. 1 zu 3 Millionen gesunken war (Signifikanz von 5 Sigma). ${ }^{* 60}$ Auf diesen Maßstab haben sich die Teilchenphysiker verständigt. Freilich können neue Messergebnisse eine physikalische Theorie jederzeit wieder in Frage stellen.

In Bezug auf Tatsachenfeststellungen vor deutschen Gerichten entspricht es der höchstrichterlichen Rechtsprechung, dass für die Beweisführung „eine absolute, das Gegenteil denknotwendigerweise ausschließende und von niemandem anzweifelbare Gewissheit nicht erforderlich ist, vielmehr ein nach der

56 Kant, Immanuel, Prolegomena zu einer jeden künftigen Metaphysik, Hamburg 1969 (Nachdruck), § 13 Anm. II.

57 Vgl. Putnam, Hilary, Vernunft, Wahrheit und Geschichte, Frankfurt a.M. 1990, 1. Kapitel.

58 Vgl. Sautoy, Marcus, Die Musik der Primzahlen. Auf den Spuren des größten Rätsels der Mathematik, München 2003.

59 Ribenboim, Paulo, Meine Zahlen, meine Freunde, Berlin, Heidelberg 2009, S. 76.

60 Vgl. Vaas, Rüdiger, Higgs, Higgs, Hurra, Bild der Wissenschaft 11/2012, S. 50. 
Lebenserfahrung ausreichendes Maß an Sicherheit genügt, das vernünftige Zweifel und nicht bloß auch denktheoretische Möglichkeiten ... nicht zulässt.”*61 Das Argument, es sei keine Außenwelt zu beweisen, erst recht keine Straftat, würde vor Gericht nicht überzeugen. Hintergrund für diesen Beweismaßstab ist der mit einer Gerichtsentscheidung verfolgte Zweck. Würden die Gerichte bei der Beweiswürdigung einen skeptischen erkenntnistheoretischen Maßstab anlegen, wäre ein Beweis nicht zu erbringen. Staat und Rechtsordnung brächen zusammen.

Nach dem Vorangegangen steht fest, dass Beweismaßstäbe von ihrer Funktion abhängig sind und unterschiedlich ausgestaltet sein können. Wie sie beschaffen sind, haben Mathematiker, Teilchenphysiker und Juristen für ihr jeweiliges Fachgebiet festgelegt. Diese Festlegungen sind menschengemacht. Es gibt keinen plausiblen Grund für die Annahme, dass es in Bezug auf Gerechtigkeitsprinzipien anders sein sollte. Allerdings haben sich die Metaethiker im Unterschied zu Mathematikern, Teilchenphysikern und Juristen auf einen für ihr Fachgebiet spezifischen Beweismaßstab bislang nicht einigen können.

\section{b) Beweismaßstab für die Begründung der westlichen Werte}

Aus der Funktion der Gerechtigkeit als dem anzustrebenden Idealzustand einer Rechtsordnung lassen sich gewisse Vorgaben für den anzulegenden Beweismaßstab gewinnen:

(1) Es muss möglich sein, Gerechtigkeitsprinzipien in geltendes Recht zu übertragen (Sollen impliziert Können, ${ }^{* 62}$ ultra posse, nemo obligatur). Dabei scheiden alle nichtrealisierbaren Überlegungen aus, was den Kreis der in Betracht kommenden Gerechtigkeitsprinzipien einengt.

(2) Gerechtigkeitsprinzipien sind mit denkbaren Alternativen zu vergleichen. ${ }^{* 63}$ Die Alternative, für welche die besseren Argumente sprechen, verdient jeweils den Vorzug. Soweit auf internationaler oder innerstaatlicher Ebene eine bessere Alternative existiert, ist der Idealzustand nicht erreicht. Dies vereinfacht die Argumentation. Es ist nicht notwendig, die absolute Richtigkeit eines Gerechtigkeitsprinzips zu beweisen, sondern lediglich seine relative Vorzugswürdigkeit.

(3) Theoretische Zweifel sind für die Bewertung der Gerechtigkeitsprinzipien ebenso belanglos wie für Tatsachenfeststellungen vor Gericht. Wird der Anspruch auf praktische Umsetzbarkeit erhoben, hat man einen geringeren Überzeugungsgrad zugrunde zu legen, wie ihn der Bundesgerichtshof für die Tatsachenfeststellung vor Gericht genügen lässt. Andernfalls gelangt man nicht zu belastbaren Ergebnissen. Weltweite Verbreitung von Gerechtigkeitsprinzipien lässt sich daher fordern, soweit kein vernünftiger Zweifel daran besteht, dass sie gegenüber Alternativen auf internationaler und innerstaatlicher Ebene vorzugswürdig sind.

(4) Eine einzelne Prämisse („eine sichere Quelle der Erkenntnis”) reicht nicht aus, um aus ihr Gerechtigkeitsprinzipien herzuleiten, wie sie für den Aufbau einer modernen Rechtsordnung erforderlich sind. ${ }^{* 64}$ Vielmehr ist wie bei Tatsachenfeststellungen vor Gericht eine Würdigung der Gesamtumstände vorzunehmen.

(5) Um das Gerechtigkeitsniveau*65 eines Rechtsprinzips oder einer Rechtsnorm zu bestimmen, ist eine Würdigung aller Anknüpftatsachen vorzunehmen. Dies hat Ähnlichkeit mit dem juristisch/ politologischen Legitimations-Konzept. Danach ergibt sich aus den Gesamtumständen (,plurale Legitimation"*66) ein Legitimationsniveau. Dieses kann sich insbesondere aus dem (mutmaßlichen) Willen der Bevölkerung (Input-Legitimation) und/oder dem Nutzen für die Bevölkerung (OutputLegitimation) ergeben. ${ }^{* 67}$ In vergleichbarer Weise ist das Gerechtigkeitsniveau zu bestimmen.

61 BGH, Urteil vom 6.11.1998, 2 StR 636/97.

62 Hepfer, Karl, Philosophische Ethik, Freiburg 2008, S. 34.

63 Niemann, Hans-Joachim, Wie objektiv kann Ethik sein, Aufklärung und Kritik, Sonderheft 5/2001, S. 23.

64 Vgl. Albert, Hans, Die Idee der kritischen Vernunft. Zur Problematik der rationalen Begründung und des Dogmatismus, Aufklärung und Kritik 2/1994, S. $16 f f$.

65 Den Begriff verwenden z.B. Höffe, Otfried, Demokratie im Zeitalter der Globalisierung, München 1999, S. 326; Losch, Bernhard, Kulturfaktor Recht. Grundwerte - Leitbilder - Normen, Köln 2006, S. 196; v.Wartenberg, Ludolf/Haß, Hans-Joachim, Investition in die Zukunft. Wie Deutschland Anschluss an die globalisierte Welt findet, Weinheim 2005, S. 181.

66 Schliesky, Utz, Souveränität und Legitimität von Herrschaftsgewalt, Tübingen 2004.

67 Sotirios, Petrovas, Parliamentary Democracy in the Lisbon Treaty, Florida 2011, S. 40 mwN. 
Eine Aufgabe der Rechtsphilosophie liegt darin, Umstände zu identifizieren, die sich auf das Gerechtigkeitsniveau positiv, negativ oder neutral auswirken, und Kriterien zu ihrer Abgrenzung und Gewichtung auszuarbeiten. Als Vorbild kann die Abwägung unterschiedlicher Rechtsgüter bei der Auslegung der Verfassung und der EMRK dienen. Optimal wäre es, wenn sich die Metaethiker zu diesem Zweck - ähnlich wie Mathematiker, Teilchenphysiker und Juristen - auf einen einheitlichen Beweismaßstab verständigen, um die Kluft zwischen Sein und Sollen zu überbrücken. Der Umstand, dass dies bislang nicht gelungen ist, steht einer Würdigung der Gesamtheit der Anknüpftatsachen nicht entgegen.

\section{c) Anknüpftatsachen}

Im Rahmen einer Würdigung der Gesamtumstände sind insbesondere die bereits erwähnten Argumente zu berücksichtigen. Während sie für eine Letztbegründung fundamentaler Gerechtigkeitsprinzipien nicht hinreichen, vermögen sie im Rahmen einer Würdigung der Gesamtumstände volle Überzeugungskraft zu entfalten:

\section{aa) Rechtsbezogene Anknüpftatsachen}

- Das Gewaltverbot ist bereits gegenwärtig Bestandteil des Völkerrechts (Art 2 Ziff. 4 UN-Charta)

- Die AEMR lässt einen Konsens der Staatengemeinschaft in Bezug auf Demokratie, Rechtsstaat und Menschenrechte als langfristig anzustrebende Ideale erkennen

- Die Staatengemeinschaft hat diesen Konsens in der Milleniumserklärung von 2000 bekräftigt. Dies verdeutlicht, dass der Konsens fortbesteht und auch Staaten umfasst, die ihre Unabhängigkeit 1947 noch nicht erlangt hatten

- Viele Staaten haben weitere Gerechtigkeitsprinzipien mit Geltung für sich völkerrechtlich verbindlich festgeschrieben, so im UN-Zivilpakt und im UN-Sozialpakt

- Der Kern des Gewaltverbots sowie das Verbot des Völkermordes, des Sklavenhandels, der Folter und der willkürlichen Tötung gehören zum völkerrechtlichen zwingenden Recht (ius cogens) und haben höchste völkerrechtliche Bedeutung

- Einige Staatengruppen haben auf regionaler Ebene noch weitergehende Regelungen verwirklicht. Hervorzuheben ist u.a. die EMRK mit ihren Individualbeschwerdemöglichkeiten

- Die westlichen Werte knüpfen an eine lange Tradition in der Rechtphilosophie an (argumentum ad verecundiam)

- Die Verwirklichung der Gerechtigkeitsprinzipien dient der Umsetzung bereits eingegangener rechtlicher Verpflichtungen.

\section{bb) Input-Legitimation (mutmaßlicher Wille der Bevölkerung)}

- Wichtige Normen sind „im moralischen Kernbestand aller großen Weltreligionen und der von ihnen geprägten Kulturen verankert”*68

- Eine Befragung von Intellektuellen vor Verabschiedung der AEMR ergab, dass die darin enthaltenen Prinzipien im Wesentlichen in allen Kulturkreisen geteilt werden ${ }^{*} 69$

- Viele Menschen verfügen über „intuitive Kenntnis” grundsätzlicher Normen, ${ }^{*} 70$ viele akzeptieren ohne weitergehende Begründung, dass alle Menschen gleichwertig sind und deswegen einen gleichen Mindeststandard an Rechten haben sollten

- Würde sich die Weltbevölkerung unter dem „Schleier des Nichtwissens” im Sinne von Rawls“ Regeln für ein Zusammenleben geben, dürfte sich eine Mehrheit - auch wenn sich ein Konsens wohl nicht erreichen ließe - für die genannten Prinzipien aussprechen

68 Habermas, Jürgen, Zur Verfassung Europas, Berlin 2011, S. 92.

69 Liese, Andrea, Staaten am Pranger. Zur Wirkung internationaler Regime auf innerstaatliche Menschenrechtspolitik, Wiesbaden 2006, S. 62.

70 Habermas, Jürgen, Zur Verfassung Europas, Berlin 2011, S. 92. 


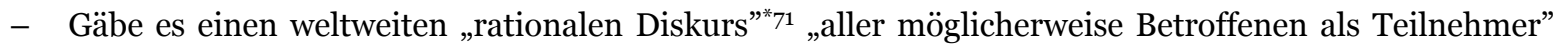
im Sinne von Habermas, würde sich die Mehrheit - auch wenn möglicherweise nicht alle Betroffenen zustimmen können - am ehesten für die genannten Werte aussprechen

- Würde die Weltbevölkerung über eine Vielzahl von Staatsmodellen abstimmen, indem sie bei jeder Abstimmung ein Modell abwählte und dann über die verbliebenen abstimmte, würde aller Voraussicht nach ein Modell übrig bleiben, das den genannten Prinzipien nahe käme.

\section{cc) Output-Legitimation (Nutzen für die Bevölkerung)}

- Die Verwirklichung der Gerechtigkeitsprinzipien trägt nach einvernehmlicher Ansicht der Staatengemeinschaft zum Frieden bei

- Sie trägt nach gleicher Ansicht zur Freiheit von Furcht und Not der Weltbevölkerung bei

- Sie erhöht die Zufriedenheit der Bevölkerung, was in Umfragen und Indizes, etwa dem Human Development Index, ${ }^{*} 72$ zum Ausdruck kommt. Darin schneiden Nordamerika, Westeuropa, Australien, Neuseeland, Japan und Südkorea besonders gut ab. Menschen mit höherem Anteil an demokratischer Partizipation sind glücklicher als Menschen in Gebieten mit weniger hohen Standards ${ }^{*} 73$

- Staaten, die westliche Werte weitgehend verwirklicht haben, etwa die USA, Kanada, die EU-Staaten, Australien und Neuseeland, genießen in der Staatengemeinschaft tendenziell hohes Ansehen. Staaten wie Iran, Syrien oder Weißrussland, in denen massive Verstöße gegen die genannten Prinzipien an der Tagesordnung sind, genießen tendenziell weniger Ansehen

- Es gibt eine gewisse Korrelation zwischen dem Fehlen von Gerechtigkeitsprinzipien und dem Scheitern von Staaten, was im failed state-Index zum Ausdruck kommt. ${ }^{*} 74$ Aus dieser Korrelation folgt keine Anleitung zum State Building. Doch ist daran u.a. ablesbar, dass das vergleichsweise demokratische und rechtsstaatliche Indien („borderline”) stabiler ist als die kommunistische VR China (,in danger”)

- Wenn die westlichen Werte in Frage gestellt werden, erfolgt dies oft von Personen, die erkennbar eigennützige Interessen (Machterhalt) verfolgen.

\section{dd) Gegenargumente}

- Es besteht in der Staatengemeinschaft kein Konsens über die konkrete Auslegung der genannten Gerechtigkeitsprinzipien. Doch ist dies in innerstaatlichen verfassungsrechtlichen und verfassungspolitischen Debatten nicht anders. Ggf. hat ein internationales Gericht über Auslegungsstreitigkeiten zu befinden

- Es besteht keine Einigkeit über den Zeitpunkt oder die Voraussetzungen, unter denen die avisierten Ziele zu verwirklichen sind. Doch ist eine Einigung über das Ob und das Wie wichtiger als über das Wann

- Es gibt immer wieder Verstöße gegen die genannten Prinzipien, selbst gegen völkerrechtliches zwingendes Recht. Doch nennt die Staatengemeinschaft solche Verstöße immer wieder beim Namen.

\section{Schlussbetrachtung}

Die genannte Auflistung ließe sich um weitere Argumente und Gegenargumente ergänzen. Zudem könnte eine Differenzierung nach einzelnen Rechtsprinzipien und Staaten erfolgen. In jedem Fall zu beachten bleibt: Nur wenn eine Würdigung der Gesamtumstände zu dem Ergebnis führt, dass kein vernünftiger Zweifel daran besteht, dass das Gerechtigkeitsniveau eines Prinzips höher ist als bei allen internationalen und innerstaatlichen Alternativen, ist die Forderung nach weltweiter Einführung gerechtfertigt.

\footnotetext{
Habermas, Jürgen, Faktizität und Geltung, Frankfurt a.M. 1992, S. 138.

United Nations Development Programme (UNDP), Human Development Report 2011, New York 2011.

3 Frey, Bruno S./Stutzer, Alois, Happiness, Economy and Institutions, in: The Economic Journal, 110 (466, Oktober), 2000, S. 918 ff; zitiert nach http://e-collection.library.ethz.ch (Stand: 18.7.2012). - DOI: http://dx.doi.org/10.1111/14680297.00570.

74 Fund for Peace/Foreign Policy (Hrsg.), The Failed State Index 2011, www.foreignpolicy.com (Stand: 19.6.2012).
} 
Das Gerechtigkeitsniveau der westlichen Werte ist angesichts der dargelegten Anknüpftatsachen hoch. Vielfach ist keine Alternativnorm ersichtlich, die ein ansatzweise vergleichbares Gerechtigkeitsniveau erreicht. So ist die Auffassung, das Verbot des Völkermordes sei ungerecht, unvertretbar. Dass nach dem hier vertretenen Ansatz Ansichten unvertretbar sein können, zeigt eine Analogie zu Tatsachenfeststellungen vor Gericht: Wenn ein Täter vor den Augen einer Menschenmenge und vor laufenden Kameras ein Verbrechen begeht, auf frischer Tat gefasst wird und die Tat gesteht, ist der Beweis unzweifelhaft erbracht. Die Ansicht, die Tat sei nicht erwiesen, weil der Beweis einer Außenwelt nicht erbracht sei oder die Tat von Außerirdischen vorgetäuscht sein könnte, erscheint unvertretbar, selbst wenn sie denklogisch nicht auszuschließen ist. Zu viele Anknüpftatsachen erhärten die Täterschaft. Ähnlich verhält es sich bei der Bewertung des Verbots von Völkermord und anderen fundamentalen Gerechtigkeitsprinzipien.

Über Bedeutung und Gewichtung einzelner Anknüpftatsachen lässt sich streiten. Der hier vertretene Ansatz wird daher in manchen Fällen zu keinem eindeutigen Ergebnis führen. Sowohl rechtlich als auch moralisch umstritten wird beispielsweise die Frage bleiben, ob und unter welchen Bedingungen die Staatengemeinschaft der Verletzung von Menschenrechten ggf. durch eine humanitäre Intervention begegnen darf. Gleiches gilt für die Frage, ob in extremer Ausnahmesituation Folter zulässig ist, ${ }^{*} 75$ etwa wenn ein Terrorist in einer Millionenstadt eine mit Zeitzünder versehene Atombombe versteckt hält und das Versteck nicht preisgibt. Denkbar sind ferner Streitigkeiten über die Reihenfolge der Einführung der genannten Werte. So besteht nach Sharp die Gefahr, dass die Abkehr von einer Diktatur ohne vorherigen Aufbau einer Zivilgesellschaft einen Rückfall in eine neue Diktatur zur Folge haben kann. ${ }^{*}{ }^{6}$ Möglicherweise erscheint es daher im Einzelfall geboten, zunächst die Zivilgesellschaft zu fördern und erst danach demokratische Schritte einzuleiten. In solchen Fällen lässt sich mit dem hier verfolgten Ansatz nicht ohne weiteres eine Zustimmung zu einer bestimmten Ansicht gewinnen. Indes ist dies kein Mangel des Ansatzes. Es kommt die Alltagserfahrung zum Tragen, dass zu bestimmten Positionen unterschiedliche Ansichten vertreten werden und vertretbar sind. Insoweit delegiert der Ansatz die Ausbalancierung der Gerechtigkeitsprinzipien auf die Staatengemeinschaft, den innerstaatlichen Gesetzgeber und die Rechtsprechung.

Das Gerechtigkeitsniveau grundlegender Werte kann einen unterschiedlichen Rang einnehmen. So ist das Verbot von Völkermord bedeutsamer als das Recht, sich an den Künsten zu erfreuen (Art. 27 Abs. 1 AEMR). Denn für das Verbot von Völkermord lassen sich gewichtigere Anknüpftatsachen anführen.

Die Bedeutung der jeweiligen Prinzipien kann sich im Laufe der Zeit ändern. Neue Rechte können zum Katalog der bestehenden hinzutreten. Andere können eine geringere Rangstelle einnehmen oder aus ihm herausfallen.

Das Gerechtigkeitsniveau von Gewaltverbot, Demokratie, Rechtsstaat und Menschenrechten ist angesichts der Vielzahl von Anknüpftatsachen außerordentlich hoch. Alternativnormen werden ein vergleichbares Gerechtigkeitsniveau im Regelfall nicht erreichen. Aus diesem Grund sollten wir an der Universalität der westlichen Werte festhalten.

Vgl. Dreier, Horst, Grundgesetz, Kommentar, Bd. 1, 2. Aufl. 2004, Art. 1 Abs. 1 Rn. 132 ff mwN.

76 Sharp, Gene, Von der Diktatur zur Demokratie. Ein Leitfaden für die Befreiung, München 2008. 